\title{
Metastatic Adenocarcinoma to the Breast arising from a Mediastinal Mature Teratoma with Somatic Malignancy: A Case Report with a Review of the Literature
}

Gwenaëlle ARHANT ( $\nabla$ arhant.gwenaelle@gmail.com )

Hôpital Charles Nicolle https://orcid.org/0000-0002-5705-5403

Dahlia Mirdad

Hopital Charles Nicolle

Anca Berghian

Centre Henri Becquerel

Nicolas PITON

Hopital Charles Nicolle

Jean-Cristophe Sabourin

Hopital Charles Nicolle

\section{Case Report}

Keywords: Mature teratoma, Malignant transformation, Metastasis, Somatic-type malignancies, Mediastinal, Breast

Posted Date: December 28th, 2020

DOI: https://doi.org/10.21203/rs.3.rs-135224/v1

License: (c) (i) This work is licensed under a Creative Commons Attribution 4.0 International License.

Read Full License 


\section{Abstract \\ Background}

Malignant transformation in mature teratomas, especially somatic-type malignancies, are extremely rare phenomena with few cases described in the literature. In the mediastinum, these lesions are often accompanied by metastases, ultimately unmasking them.

\section{Case presentation}

We report an exceptional case of metastatic adenocarcinoma to the breast arising from a mediastinal mature teratoma with malignant transformation in a 31-year-old female. The patient initially presented with a breast lesion that was later diagnosed as either a triple negative ductal carcinoma or a metastatic adenocarcinoma of unknown origin. Extensive clinical workup and radiological imagining revealed an anterior mediastinal mass. Subsequent pathological examination of the mass showed a mature teratoma with malignant transformation equivalent to that found in the breast biopsy.

\section{Conclusion}

Mediastinal teratomas with malignant transformation (TMTs) are rare entities; however these tumors should be suspected in cases of suspicious mediastinal masses presenting with unusual clinical and radiological signs, such as metastasis of unknown primary origin. Pathological corroboration of extensive sampling and meticulous microscopic examination is crucial for the diagnosis in order to tailor subsequent treatment.

\section{Background}

Breast cancer accounts for more than $20 \%$ of all cancers affecting women (1). Conversely, metastases to the breast are rare and represent only $0.2 \%$ to $1.3 \%$ of mammary tumours (2-4). Among occult cancers, $30 \%$ are initially presented by breast metastasis (3-5). Imaging studies usually reveal a single welldefined, occasionally speculated mass. The most common origin of these metastases are controlateral breast cancers, however, they can also arise from other neoplasms such as: carcinomas from the lung and ovary, melanomas, lymphomas and rhabdomyosarcomas particularly seen in young female patients $(5,6)$. However, many other primitive sites have been reported $(7)$.

Here we report a highly unusual case of metastatic adenocarcinoma to the breast in a young female arising from a mediastinal mature teratoma with malignant transformation.

\section{Case Presentation}


A previously healthy 31-year-old woman was referred to our cancer center following a recent discovery of a left breast lump. Her family history included an aunt who had died from ovarian cancer. Clinical examination revealed a nodular lesion located at the exterior upper quadrant of the left breast with no signs of inflammation or lymph node involvement. Routine hematological and biochemical investigations were unremarkable. Ultrasound study and mammography both showed a mass measuring $37 \mathrm{~mm}$, classified as ACR4 (8). Biopsy of the mass revealed mammary parenchyma diffusely infiltrated by a moderately differentiated adenocarcinoma composed of variable sized glands in a discreetly fibrous stroma. These glands were lined by tall columnar epithelial cells displaying abundant eosinophilic cytoplasm, moderate atypia and rare mitoses. Some cells had intra-cytoplasmic mucin vacuoles (Figures 1 and 2).

An extensive immunohistochemical panel was performed and showed positivity with epithelial markers: CK7+ (strong and diffuse); CK20+ and CEA + (weak and focal).

Other stains such as: Oestrogen Receptor (ER), Progesterone Receptor (PR), HER2, Mammaglobin, GATA3, TTF1, P40, P63, CD117 and PAX8 were all negative (Figure 3).

The initial diagnosis proposed was a triple negative grade 1 invasive ductal carcinoma according to the modified Scarff-Bloom-Richardson classification system (9).

Consequent imaging by Computed Tomography revealed a well-defined cystic anterior mediastinal mass with central necrosis, measuring about $10 \mathrm{~cm}$ and compressing adjacent anatomical structures. It also showed atelectasis, pulmonary and pleural thickening, multiple pulmonary nodules as well as regional lymphadenopathy.

Scintigraphy revealed multiple bone foci at the levels of $L 3, S 1$ and $S 2$ and at the level of the 6th intercostal rib.

A Positron-emission tomography scan found two suspicious hypermetabolic lesions in the left breast, multiple positive foci at the level of the left pleura, left lung, left adrenal gland and multiple bone sites associated with mediastinal lymphadenopathy. No anomalies in the gastrointestinal tract were found.

Subsequent biopsy of the mediastinal mass revealed a moderately differentiated adenocarcinoma similar in morphology to that found in the breast biopsy. Interestingly, the immunohistochemical study showed a strong positivity for CK8/18 and CK7, a focal positivity for CEA and a mitotic index of $60 \%$. All other immunostains including CK20, CDX2 and breast tissue markers were negative. Based on these findings, a left anterior thoracotomy was performed to remove the mediastinal lesion.

On gross examination, the resected tumor measured $12 \times 10 \times 7 \mathrm{~cm}$ and weighed $99 \mathrm{~g}$. It was well demarcated with no obvious signs of rupture. No definite capsule or lung parenchyma were identified. The external surface had a glistened variegated appearance with alternating yellowish and congested areas. On sectioning, the mass was unilocular with a predominantly smooth wall and contained grumous material with hair. 
Multiple thickened bourgeon areas measuring up to $1.5 \mathrm{~cm}$ associated with small yellowish colored granules were discovered inside the wall and on its interior surface. No papillary excrescences were found.

Microscopically, the cystic wall consisted of fibrocollagenous tissue lined by cuboidal and/or tall columnar mucin secreting epithelium with areas of minimal chronic inflammation and vascular congestion (Figure 5). On sectioning of the thickened areas, mature elements of all three germ layers were found:

- mesodermal derivatives such as adipose tissue, cartilage, and muscle bundles,

- endodermal derivatives such as gastrointestinal tract tissue and ciliated bronchial tissue

- and ectodermal derivatives such as stratified squamous epithelium and sebaceous glands.

There were no immature teratomatous elements or other germ cell components associated. Furthermore, we noted multiple foci of atypical cells, arranged in groups or glands and surrounded by a reactive fibrous stroma. These cells contained abundant eosinophilic cytoplasm, sometimes with intracytoplasmic vacuoles and displayed a moderate degree of pleomorphism and atypia (Figure 5). The immunohistochemical profile was similar to that found in the breast biopsy and showed a positivity for cytokeratines, BerEP4 and CDX2, suggesting a digestive origin (Table 1).

A final diagnosis of a mature teratoma with somatic-type malignant transformation, most likely an adenocarcinoma, with secondary breast metastasis was concluded.

The patient's postoperative course was uneventful and she was subsequently discharged. Unfortunately, the patient died a few months later.

\section{Discussion And Conclusion}

Triple-negative ductal carcinomas account for only $15 \%$ of mammary tumours but are responsible for a disproportionate number of deaths. In fact, these tumours are most often of high grade; low grades (or grade I) represent only 9.8 to $15.1 \%$ of these cases $(10,11)$. Due to the overall rarity of this type of tumour, it is necessary to be cautious when establishing this diagnosis by thoroughly eliminating all other differentials, including metastasis. This case reports a metastatic adenocarcinoma to the breast in a young female arising from a mediastinal mature teratoma with somatic-type malignant transformation. Teratomas belong to germ cell tumours (GCTs), divided into: seminomas, malignant "non-seminomatous GCTs" (such as embryonal carcinoma, yolk sac tumours, choriocarcinoma and mixed germ cell tumors), and teratomas both mature and immature. Mediastinal teratomas account for 50 - $70 \%$ of all mediastinal GCTs and 7 to $9,3 \%$ of mediastinal tumours (12).

According to the most recent World Health Organization definition, a teratoma with somatic-type malignancy is a teratoma containing one or more components of a non-germ cell malignant tumour, 
which may be a sarcoma or a carcinoma. These tumours represent 10 to $20 \%$ of all mediastinal GCTs with somatic-type malignancies (12). Histologically, it seems that the malignant component is often carcinomatous $(13,14)$ but this somatic-type only represents $37 \%$ of all GCTs with somatic-type malignancies (15). These tumours mostly always occur in males (12), between the ages of 20 to 40 years; however a few cases concerning females have been reported in the literature, mainly as case reports $(14,16-22)$.

Same clinical features are also used to classify these tumours into two categories: TMTs induced by chemotherapy and/or irradiation and naturally occurring TMTs $(14,16,18)$. Most reported cases have been from the former group, chiefly occurring in young patients with an initial presentation of a malignant germ cell tumour. On the other hand, naturally occurring TMTs in the mediastinum are extremely rare and occur in older patients in the context of mature teratomas of long standing duration, generally with good prognoses $(14,16)$. In our case, the malignant transformation most likely occurred naturally due to the absence of chemotherapy or radiation exposure. Contrarily, the breast metastasis demonstrates an aggressive nature to this tumour. To our knowledge, this is the first reported case of a mediastinal TMT unmasked by breast metastasis.

TMTs should be suspect under certain clinical and radiological presentations such as: a voluminous mediastinal tumour measuring at least $10 \mathrm{~cm}$ in size (14), adhesion to surrounding structures with or without invasion, local wall thickening with fatless solid areas and a contrast-enhancing capsule with a rough and uneven internal surface $(13,22)$.

These non-germ cell malignancies are postulated to originate either from totipotential germ cells differentiating to somatic type cells or from pre-existing mature teratoma elements to non-germ cell malignancy $(14,20,22)$. Molecular studies have shown an isochromosome 12p (i[12p]) genotype with no clearly defined consequence in carcinogenesis, that is shared by both the somatic-type malignancy and its associated germ cell tumor component (12). The latter hypothesis is favored by the finding that "pure" mature mediastinal teratomas show no chromosome 12 abnormalities while a shared $i(12 p)$ abnormality is characteristic of teratomas that are clonally related with somatic type malignancies.

Interestingly, the majority of somatic-type malignancies do not immunohistochemistraly express PLAP, AFP, beta-hCG, or CD30, which are normally expressed in GCTs, but rather stain like their counterparts found elsewhere in the body. Cytokeratin immunostains, such as CK7 and CK 20, can be used to distinguish adenocarcinomas from other types of malignancies (21).

According to the literature, prognosis is poor compared to other GCTs, and depends mainly on surgical resection which is currently the recommended treatment. Other factors include, the primary tumor site and its margins, the type of transformed histology and the number of prior chemotherapy regimens before the development of TMT (23). Due to its rarity, the treatment of these tumors does not have a standardised chemotherapy protocol. However, adapted chemotherapy based on the TMT somatic malignant component is hypothesized to improve the overall outcome of these patients $(13,24-26)$. 
Despite their rarity, mediastinal TMTs should be suspected in all clinically diagnosed cases of mediastinal mature cystic teratomas with unusual metastasis. Unfortunately, clinical and radiological examinations cannot always predict the malignant component of these tumors. Therefore, pathological corroboration with extensive sampling and meticulous microscopic examination is crucial for accurate diagnosis, and for a tailoring subsequent treatment.

\section{Abbreviations}

- TMTs : teratomas with malignant transformation

- GCTs : germ cell tumours

- CK : Cytokeratin

- ER : Oestrogen Receptor

- PR : Progesterone Receptor

- CEA : carcinoembryonic antigen

\section{Declarations}

\section{Ethics approval and consent to participate}

Ethical approval was obtained according to the agreement of the tumor biobank of Rouen University Hospital (tissue sample collection $n^{\circ}$ DC2008-689) by the institutional review board of Rouen University Hospital and the French Ministry of Scientific Research.

\section{Consent for publication}

Not applicable

\section{Availability of data and materials}

All data supporting the conclusions of this article is included within the article.

\section{Competing interests}

The authors declare that they have no conflicting interests

\section{Funding}

Not applicable

\section{Authors' contributions}

Gwenaëlle ARHANT and Dahlia MIRDAD equally contributed to this work, they wrote the article and reviewed the literature. 
Anca BERGHIAN and Nicolas PITON diagnosed the case.

Nicolas PITON and Jean-Christophe SABOURIN revised the article.

\section{Authors' information}

Gwenaëlle Arhant* - CHU de Rouen, 1 rue de Germont, 76031 Rouen cedex

Dahlia Mirdad* - CHU de Rouen, 1 rue de Germont, 76031 Rouen cedex

*these authors contributed equally to this work

\section{References}

1. Ferlay J, Soerjomataram I, Dikshit R, Eser S, Mathers C, Rebelo M, et al. Cancer incidence and mortality worldwide: sources, methods and major patterns in GLOBOCAN 2012. Int J Cancer. 1 mars 2015;136(5):E359-386.

2. Hajdu SI, Urban JA. Cancers metastatic to the breast. Cancer. juin 1972;29(6):1691-6.

3. Alvarado Cabrero I, Carrera Alvarez M, Pérez Montiel D, Tavassoli FA. Metastases to the breast. Eur J Surg Oncol. déc 2003;29(10):854-5.

4. Lee AHS. The histological diagnosis of metastases to the breast from extramammary malignancies. J Clin Pathol. déc 2007;60(12):1333-41.

5. Georgiannos SN, Chin J, Goode AW, Sheaff M. Secondary neoplasms of the breast: a survey of the 20th Century. Cancer. 1 nov 2001;92(9):2259-66.

6. Troxell ML. Merkel cell carcinoma, melanoma, metastatic mimics of breast cancer. Semin Diagn Pathol. sept 2017;34(5):479-95.

7. DeLair DF, Corben AD, Catalano JP, Vallejo CE, Brogi E, Tan LK. Non-mammary metastases to the breast and axilla: a study of 85 cases. Mod Pathol. mars 2013;26(3):343-9.

8. Mercado CL. BI-RADS update. Radiol Clin North Am. mai 2014;52(3):481-7.

9. Le Doussal V, Tubiana-Hulin M, Friedman S, Hacene K, Spyratos F, Brunet M. Prognostic value of histologic grade nuclear components of Scarff-Bloom-Richardson (SBR). An improved score modification based on a multivariate analysis of 1262 invasive ductal breast carcinomas. Cancer. 1 nov 1989;64(9):1914-21.

10. Gonzalez-Angulo AM, Timms KM, Liu S, Chen H, Litton JK, Potter J, et al. Incidence and outcome of BRCA mutations in unselected patients with triple receptor-negative breast cancer. Clin Cancer Res. 1 mars 2011;17(5):1082-9.

11. Dent R, Trudeau M, Pritchard KI, Hanna WM, Kahn HK, Sawka CA, et al. Triple-negative breast cancer: clinical features and patterns of recurrence. Clin Cancer Res. 1 août 2007;13(15 Pt 1):4429-34.

12. Ströbel $P$, Chan JKC, Looijenga LHJ, et al. Germ cell tumours with somatic-types solid malignancy. In: Travis WD, Brambilla E, Burke AP, Marx A, Nicholson AG, eds. World Health Organization 
Classification of Tumours. Pathology and Genetics of Tumours of the Lung, Pleura, Thymus and Heart. Lyon, France: IARC Press; 2015: 263-264.

13. Cyril Habougit, Violaine Yvorel, Abdulrazzaq Sulaiman, Bachir Hag, Michel Péoc'h, Fabien Forest. Mediastinal Mature Teratoma With Malignant Carcinomatous Transformation (Somatic-Type Malignancy) With Metastatic Course. Int J Surg Pathol. 1 déc 2015;23(8):682-4.

14. Paliwal N, Gupta K, Dewan RK, Mullick S. Adenocarcinoma (somatic-type malignancy) in mature teratoma of anterior mediastinum. Indian J Chest Dis Allied Sci. mars 2013;55(1):39-41.

15. Motzer RJ, Amsterdam A, Prieto V, Sheinfeld J, Murty VV, Mazumdar M, et al. Teratoma with malignant transformation: diverse malignant histologies arising in men with germ cell tumors. J Urol. janv 1998;159(1):133-8.

16. Morinaga S, Nomori H, Kobayashi R, Atsumi Y. Well-differentiated adenocarcinoma arising from mature cystic teratoma of the mediastinum (teratoma with malignant transformation). Report of a surgical case. Am J Clin Pathol. avr 1994;101(4):531-4.

17. Gupta P, Singh S, Yadava K, Kumari N. Typical carcinoid arising in mature teratoma of anterior mediastinum. Asian Cardiovasc Thorac Ann. févr 2012;20(1):80-2.

18. Jung JI, Park SH, Park JG, Lee SH, Lee KY, Hahn ST. Teratoma with Malignant Transformation in the Anterior Mediastinum: A Case Report. Korean Journal of Radiology. 1 sept 2000;1(3):162-4.

19. Shimizu K, Nakata M, Hirami Y, Akiyama T, Tanemoto K. Teratoma with malignant transformation in the anterior mediastinum. J Thorac Cardiovasc Surg. juill 2008;136(1):225-7.

20. Agarwal S, Mullick S, Gupta K, Dewan RK. Mediastinal Teratoma with Coexisting Adenocarcinoma and Carcinoid Tumor (Somatic-Type Malignancy): A Case Report with a Review of the Literature. Turk Thorac J. avr 2015;16(2):101-4.

21. Chang Y-L, Wu C-T, Lee Y-C. Mediastinal and retroperitoneal teratoma with focal gastrointestinal adenocarcinoma. J Thorac Oncol. sept 2006;1(7):729-31.

22. Chen $\mathrm{C}$, Zheng $\mathrm{H}$, Jiang $\mathrm{S}$. An unusual case of giant mediastinal teratoma with malignant transformation. Ann Thorac Surg. juill 2008;86(1):302-4.

23. Giannatempo P, Pond GR, Sonpavde G, Albany C, Loriot Y, Sweeney CJ, et al. Treatment and Clinical Outcomes of Patients with Teratoma with Somatic-Type Malignant Transformation: An International Collaboration. J Urol. juill 2016;196(1):95-100.

24. El Mesbahi O, Terrier-Lacombe M-J, Rebischung C, Theodore C, Vanel D, Fizazi K. Chemotherapy in patients with teratoma with malignant transformation. Eur Urol. mai 2007;51(5):1306-11; discussion 1311-1312.

25. Donadio AC, Motzer RJ, Bajorin DF, Kantoff PW, Sheinfeld J, Houldsworth J, et al. Chemotherapy for teratoma with malignant transformation. J Clin Oncol. 1 déc 2003;21(23):4285-91.

26. Lin C, Du Y, Li Y, Wang H, Chang J. Superior mediastinal mature cystic teratoma with gastrointestinal adenocarcinoma transformation: Report of a case. Oncotarget. 21 juin 2016;7(25):38392-7. 


\section{Tables}

Table 1. Comparison of immunohistochemical profile of the breast and mediastinal mass.

\begin{tabular}{|lll|}
\hline Immunohistochemical Stain & Tumor Cells in mediastinum & Tumors Cells in breast lesion \\
\hline ER & Negative & Negative \\
\hline PR & Negative & Negative \\
\hline GATA 3 & Negative & Negative \\
\hline Mammoglobine & Not performed & Negative \\
\hline CK7 & Not performed & Negative \\
\hline CK20 & Positive (strong and diffuse) & Positive (strong and diffuse) \\
\hline CDX2 & Positive (weak and focal) & Negative \\
\hline BerEP4 & Positive & Positive (weak) \\
\hline TTF1 & Positive & Not performed \\
\hline Calretinin & Negative & Negative \\
\hline CEA & Negative & Not performed \\
\hline PAX 8 & Not performed & Negative \\
\hline
\end{tabular}

\section{Figures}




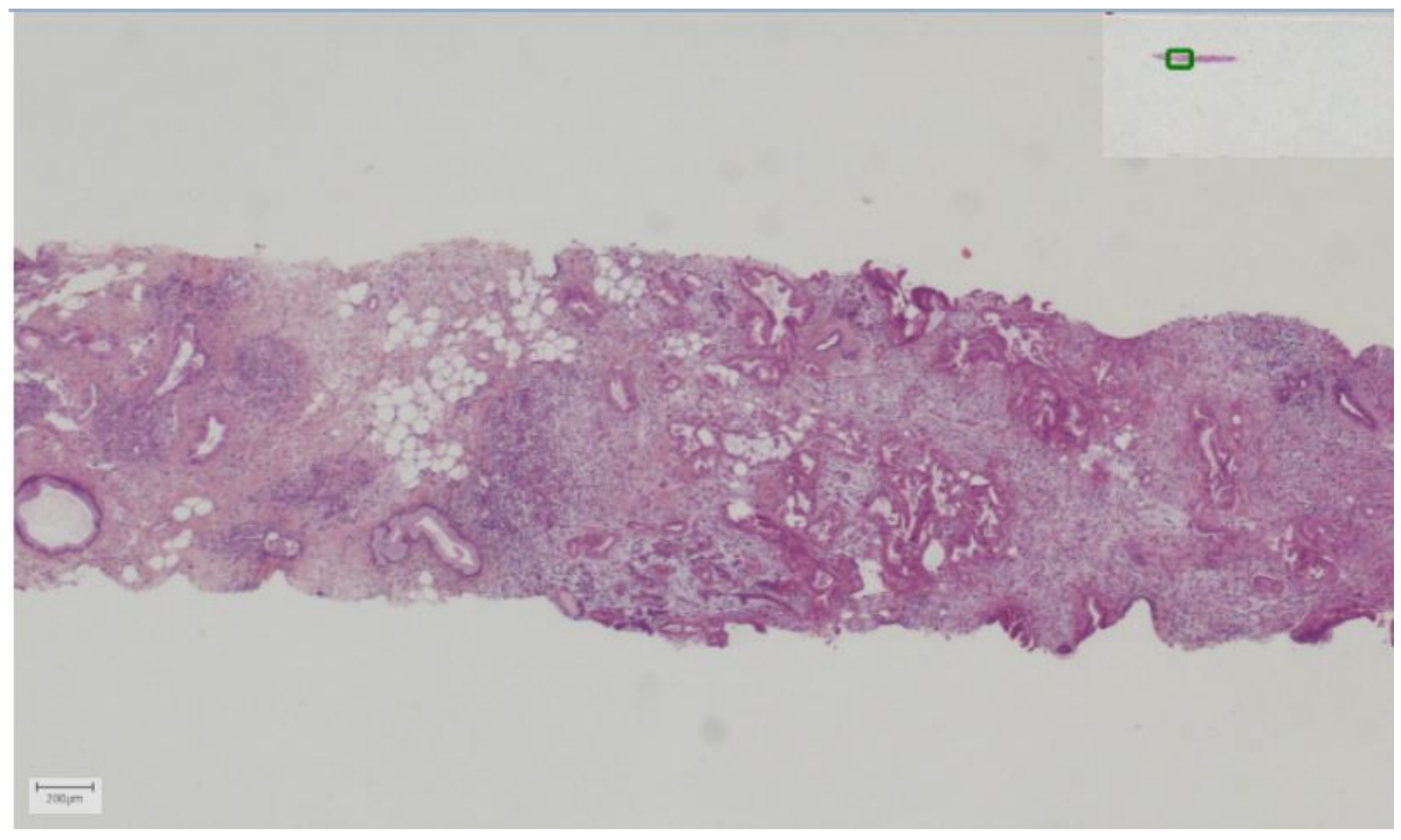

\section{Figure 1}

Tumour infiltration into the breast parenchyma with surrounding fibrous stroma. (Hematoxylin and eosin stain, X2.5).

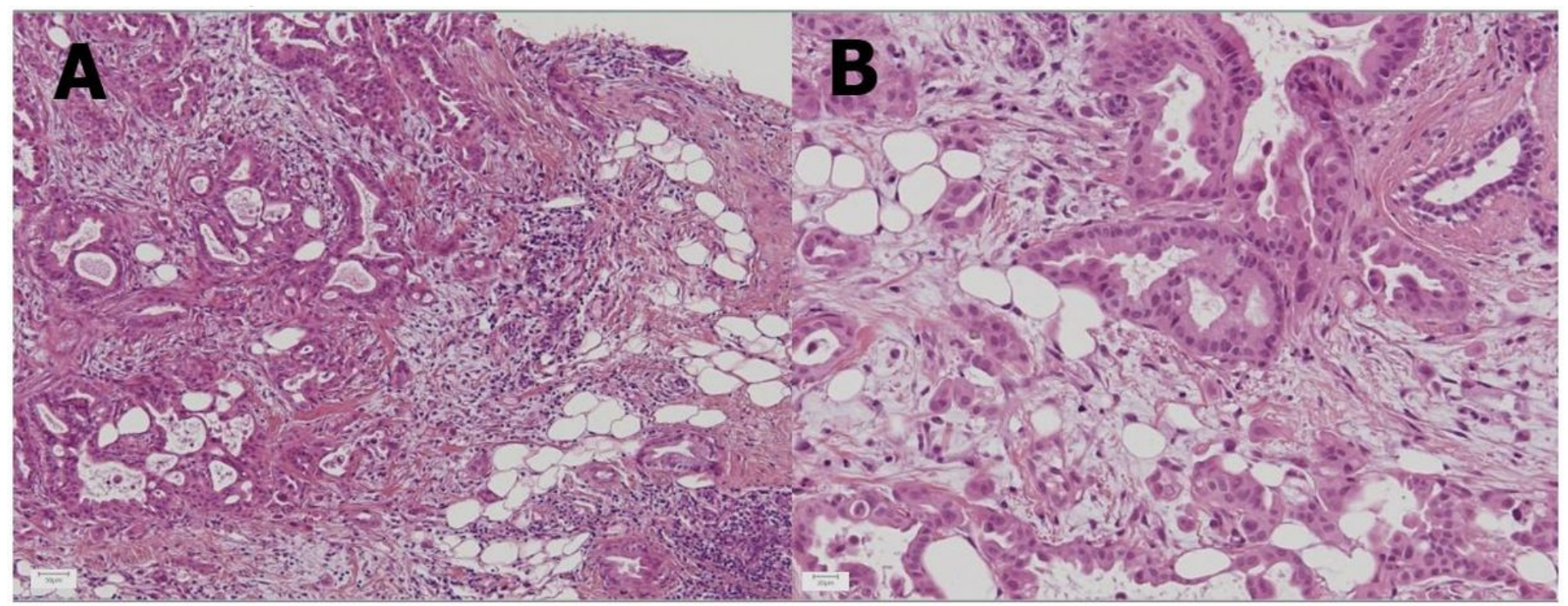

\section{Figure 2}

Tumor histology. A) Moderately differentiated adenocarcinoma composed of glands of various sizes and shapes (Hematoxylin and eosin stain, X10). B) Malignant glands are lined by columnar epithelium 
exhibiting moderate atypia (Hematoxylin and eosin stain, X20).

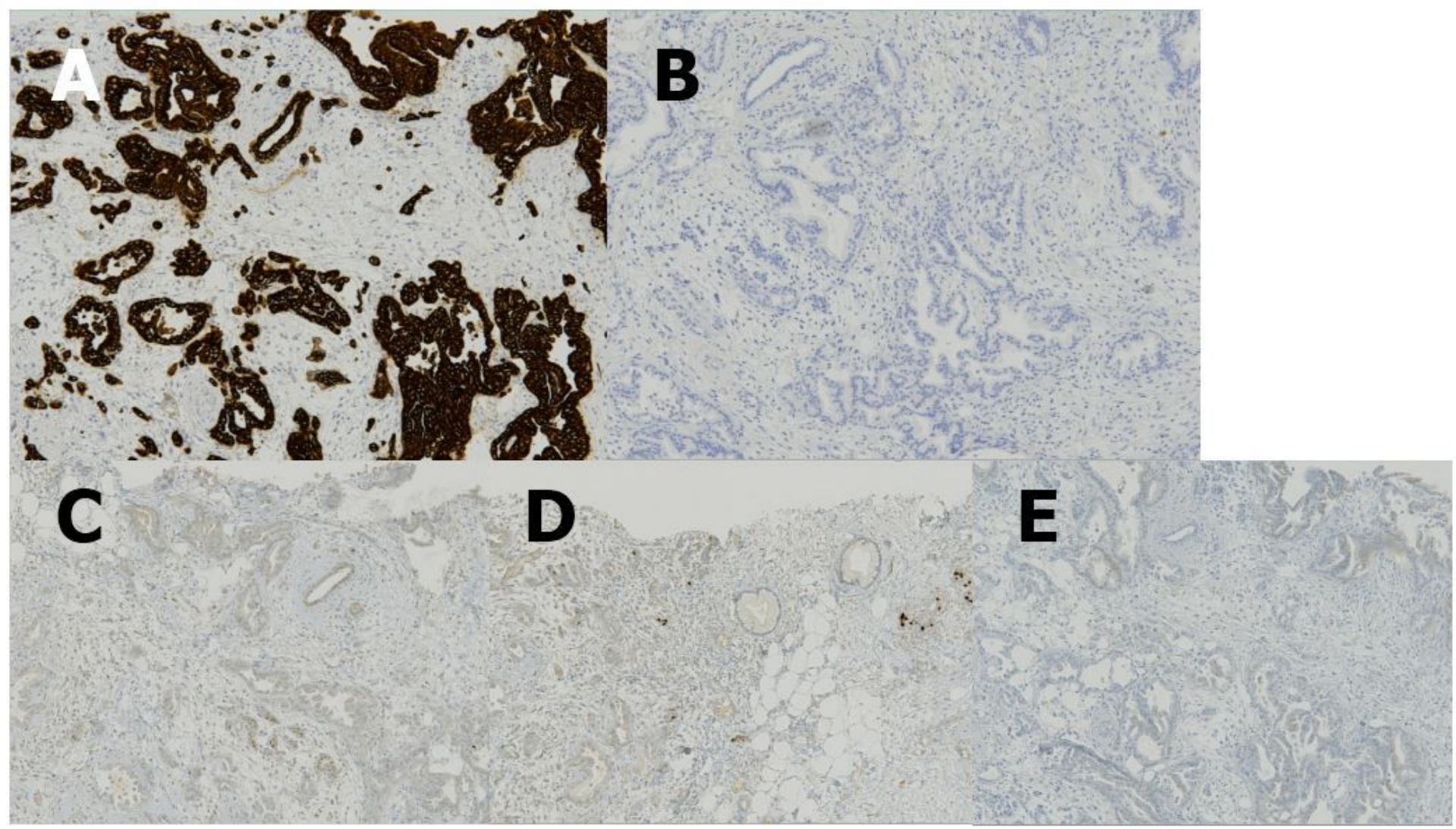

Figure 3

Immunohistochemical stains on the breast biopsy. A) CK7+ strongly and diffusely positive. B) CK20 negative. C) Triple negative (PR-, RE- and HER2-). 

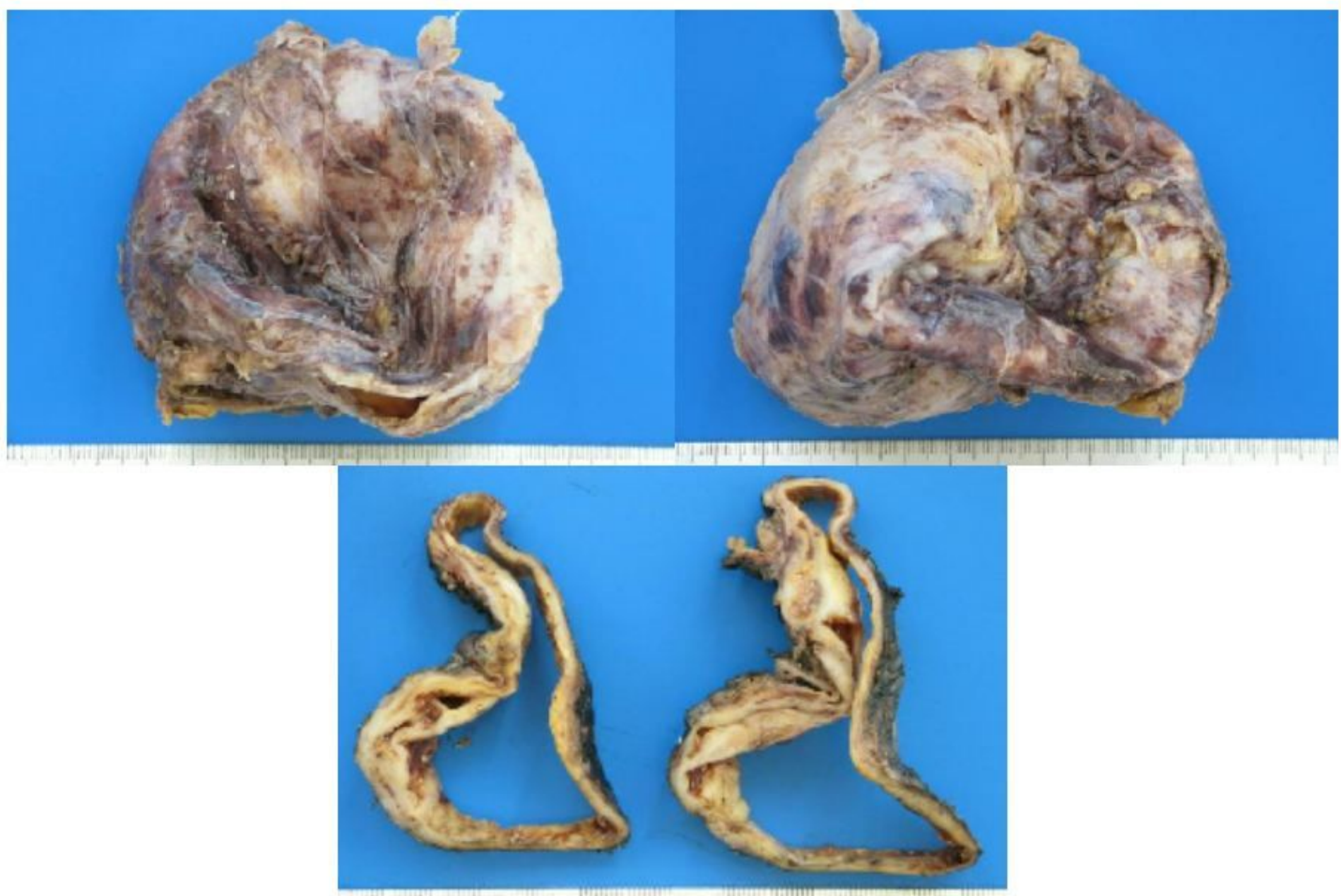

\section{Figure 4}

Macroscopic appearance of resected mediastinal mass. Well demarcated. No lung parenchyma was identified. On section, the mass was unilocular with areas of wall thickening associated with small yellow granules inside and on the interior surface of the cystic wall.

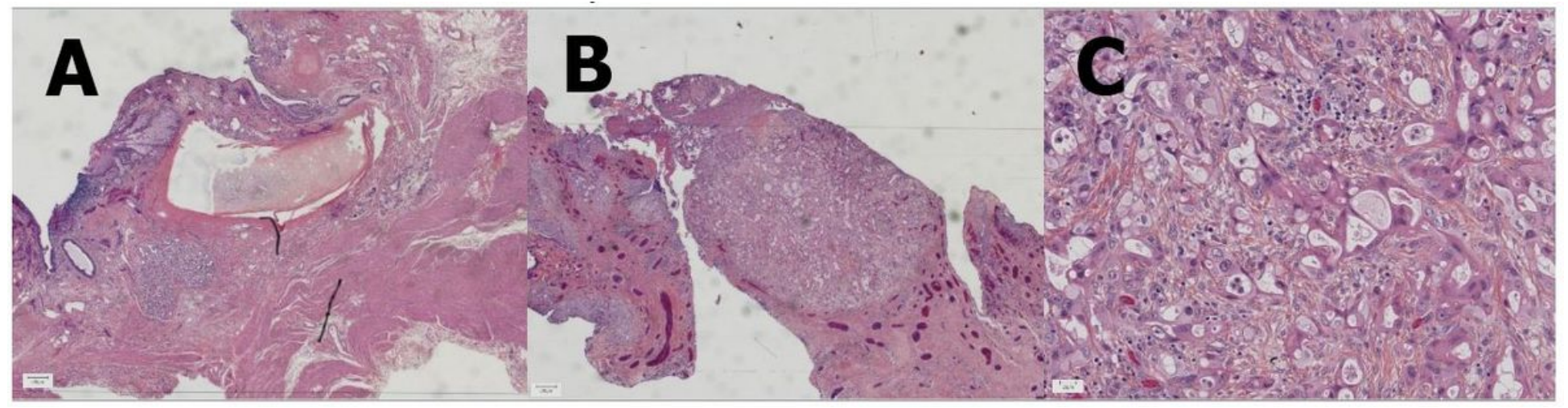

\section{Figure 5}

Tumor histology. A) Mature Teratoma Germ cell layers (Hematoxylin and eosin stain, X2.5). B and C) Mature Teratoma with Somatic Malignancy demonstrates a moderately differentiated adenocarcinoma 
(Hematoxylin and eosin stain, X2.5 and X20).

Page 13/13 